
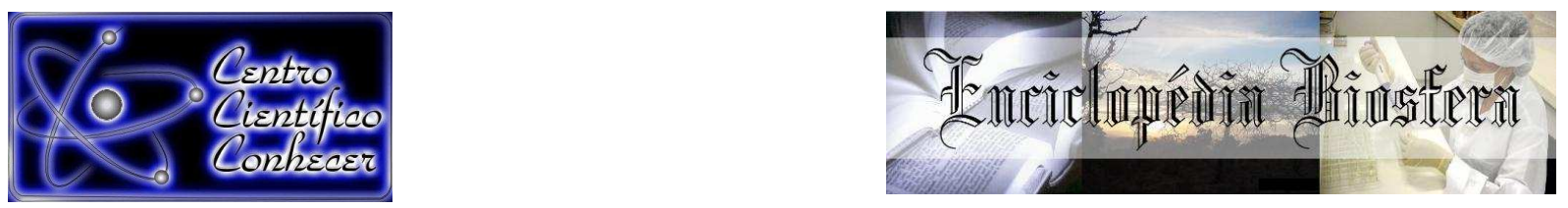

\title{
OS GAFANHOTOS ACRIDOIDEA (ORTHOPTERA: CAELIFERA) DA COLEÇÃO ZOOLÓGICA DIDÁTICO-CIENTÍFICA Dr. JOACHIM ADIS DA UNIVERSIDADE DO ESTADO DO PARÁ
}

\author{
Ana Lúcia Nunes Gutjahr ${ }^{1}$, Carlos Elias de Souza Braga² \\ 1 Professora Doutora do Departamento de Ciências Naturais da Universidade do \\ Estado do Pará (melcam@uol.com.br). Belém, Pará, Brasil. \\ 2 Professor Doutor do Departamento de Ciências Naturais da Universidade do Estado \\ do Pará, Belém, Pará, Brasil. \\ Recebido em: 03/10/2016 - Aprovado em: 21/11/2016 - Publicado em: 05/12/2016 \\ DOI: 10.18677/EnciBio_2016B_116
}

\begin{abstract}
A região Amazônica é conhecida por sua imensa diversidade biológica. Neste contexto, as coleções científicas constituem grandes centros de informação de biodiversidade, principalmente na Amazônia. A Coleção Zoológica Didático-Científica Dr. Joachim Adis, da Universidade do Estado do Pará (CZJA-UEPA), possui um acervo de invertebrados com ênfase na entomofauna regional. Neste estudo, busca-se apresentar uma lista das espécies de gafanhotos Acridoidea, bem como o mapeamento desses insetos na região amazônica. Para o estudo, os dados foram obtidos no banco de dados da CZJA. Constatou-se que o acervo da CZJA possui duas famílias de Acridoidea (Acrididae: $\mathrm{n}=$ 562; Romaleidae: $n=436$ ), oito subfamílias, 15 tribos, 30 gêneros e 42 espécies, todos oriundos de quatro estados brasileiros (Amapá, Amazonas, Maranhão e Pará). Embora o acervo da CZJA-UEPA seja pequeno e jovem (sete anos), quando comparado com acervos de grandes coleções seculares, este deve ser valorizado, não somente por sua representatividade regional, mas por reunir espécies de gafanhotos terrestres e semiaquáticos que exploram diferentes habitats em ambientes naturais como as florestas primárias e secundárias, campos, colônias de macrófitas aquáticas e antropizados como as roças, plantações agrícolas e ambientes urbanos. Tal acervo deve também ser reconhecido pelo fato de que os gafanhotos Acridoidea dessa coleção encontrarem-se catalogados, tombados e em bom nível de aferimento taxonômico, podendo possibilitar 0 desenvolvimento de estudos de diferentes áreas do conhecimento.
\end{abstract}

PALAVRAS-CHAVE: Acridofauna, Coleção Zoológica, Região Amazônica. 


\title{
THE GRASSHOPPERS ACRIDOIDEA (ORTHOPTERA: CAELIFERA) OF THE ZOOLOGICAL AND SCIENTIFIC-EDUCATIONAL COLLECTION DR. JOACHIM ADIS OF THE STATE UNIVERSITY OF PARÁ
}

\begin{abstract}
The Amazon region is known for its immense biological diversity. In this context the scientific collections constitute major centers of biodiversity information, especially in the Amazon region. The Zoological and Scientific- Didactic Collection Dr. Joachim Adis, of the State University of Pará (CZJA-UEPA), has a collection of invertebrates with emphasis on regional entomofauna. In this study, it is looked to make a list of species of grasshoppers Acridoidea as well as the mapping of these insects in the Amazon region. For the study, data were obtained from the CZJA database. It was noticed that the CZJA collection has two families of Acridoidea (Acrididae: $n=562$; Romaleidae: $n=436$ ), eight subfamilies, 15 tribes, 30 genera and 42 species, all originating from four Brazilian states (Amapá, Amazonas, Maranhão and Pará). Although the CZJA-UEPA collection is small and young (seven years) when compared to large secular collections, one should appreciate the CZJA collection, not only for regional representativeness that it has, but by collecting species of land and semi-aquatic grasshoppers occupying different habitats in natural environments such as primary and secondary forests, open fields, macrophyte communities as well as anthropic environments such as agricultural plantations and urban environments. This collection should also be recognized by the fact that the grasshoppers Acridoidea of this collection area cataloged and in a good level of taxonomic identification, which may enable the development of studies in different knowledge fields.
\end{abstract}

KEYWORDS: Acridofauna, Amazon region, Zoological collection.

\section{INTRODUÇÃO}

A região amazônica é considerada detentora da maior biodiversidade do planeta e abrange uma área de floresta correspondente a cerca de seis milhões de $\mathrm{km}^{2}$ (UNEP, 2001), dos quais, estima-se que 3,6 milhões de $\mathrm{km}^{2}$ (cerca de 60\%) estejam em território brasileiro (SOUZA, 2010). Nesse contexto, o Brasil é considerado o país detentor da maior riqueza de espécies de animais e vegetais do mundo, representando cerca de 10 a 20\% de todas as espécies descritas (HADDAD, 1998; GUEDES, 2012), o que representa uma grande parte da biodiversidade mundial.

Os centros de documentação da biodiversidade conhecidos correspondem às coleções científicas, que representam as fontes permanentes de pesquisa, extensão e ensino, bases da construção e desenvolvimento do conhecimento humano (MAGALHÃES et al., 2001; SILVA et al., 2012). As coleções biológicas podem ser classificadas em coleções didáticas (destinadas ao ensino, demonstrações e treinamentos), coleções de pesquisa (dedicadas ao desenvolvimento de estudos taxonômicos e biológicos), coleções regionais (reúnem espécimes de determinada região geográfica), coleções especiais (desenvolvidas conforme o interesse específico de estudo) e coleções de identificação (voltadas para o apoio ao reconhecimento de determinado material para diversas finalidades de estudo) (PAPAVERO, 1994).

Entre as coleções que tratam da biodiversidade, destacam-se as Coleções Zoológicas que detêm acervos que reúnem os representantes dos mais diversos grupos 
de animais, que são preparados (preservados, montados, etiquetados), classificados, identificados e organizados para atender uma variedade de estudos, tais como taxonômicos, ecológicos ou biogeográficos, ressaltando que se o acervo for didático, a importância é relevante para o ensino e a aprendizagem, seja ao nível de educação básica, graduação e pós-graduação.

As coleções zoológicas científicas e didáticas são de grande importância para o conhecimento da história evolutiva dos animais, no mapeamento da biodiversidade e no estudo da taxonomia e sistemática. Elas podem conter uma variedade de grupos animais e seus acervos podem ser utilizados para estudos, além dos mencionados anteriormente, também, biológicos, de saúde pública, entre outros. Estes tipos de coleções são normalmente encontrados em universidades, instituições de pesquisa e em museus (AURICCHIO \& SALOMÃO, 2002). Ressalta-se que uma coleção inserida no âmbito universitário tem potencialmente e características distintas de outras coleções, construídas fora das universidades (RIBEIRO, 2013).

A Coleção Zoológica Didático-Científica Dr. Joachim Adis, da Universidade do Estado do Pará (CZJA-UEPA), foi criada em 2009 a partir de um projeto que abordou a coleta de animais invertebrados pertencentes ao Filo Arthopoda. Atualmente o acervo da coleção conta com cerca de 10.000 espécimes desses animais. Seu acervo já foi apresentado em exposições durante eventos científicos (feiras de ciências e tecnologia, fórum de pesquisa, ensino, extensão e pós-graduação e feiras vocacionais) promovidos pela UEPA e também em exposições realizadas em praças públicas por ocasião de um projeto de divulgação cientifica que objetivou a popularização da ciência, intitulado "Exposição Itinerante de Biodiversidade Amazônica: A Ciência vai à praça", apoiado pelo $\mathrm{MCTI} / \mathrm{CNPq}$. Ressalta-se que a CZJA-UEPA também desempenha um papel importante na graduação por disponibilizar aos docentes o material zoológico, para às aulas práticas, através de seu acervo didático. Além de envolver estudantes de graduação e pós-graduação, em suas atividades de curadoria.

Entre os animais invertebrados que compõem 0 acervo da CZJA-UEPA encontra-se o Filo Arthropoda, as quais pertencem às Classes Diplopoda, Chilopoda, Crustácea, Arachnida e Insecta. Dentro desta última classe destacam-se os insetos pertencentes a ordem Orthoptera, cujos representantes são os gafanhotos (Acridoidea), grilos (Grylloidea), esperanças (Tettigonioidea) e manés-magros (Proscopioidea).

Os gafanhotos são insetos que possuem grande importância ecológica (NUNESGUTJAHR \& BRAGA, 2011) e econômica (NUNES-GUTJAHR \& BRAGA, 2015) em decorrência de deter espécies pragas que causam danos agrícolas. Insetos da superfamília Acridoidea representam os gafanhotos verdadeiros (NUNES-GUTJAHR \& BRAGA, 2011; 2012) que possuem como principais características o primeiro para de asas do tipo pergaminácea denominada tégmina, terceiro par de pernas adaptados para saltar e as antenas com cerca de 30 antenômeros e não ultrapassam o abdome. Esta superfamília encontra-se em melhor nível de aferimento no acervo da CZJA, visto que os espécimes estão tombados e identificados ao nível específico e encontram-se registrados no banco de dados da coleção. Por tal razão, este trabalho busca realizar um estudo diagnóstico para a compilação de uma lista das espécies de gafanhotos Acridoidea da CZJA-UEPA, bem como o mapeamento dos locais de procedência das espécies do acervo, para verificar o nível de amostragem desses insetos na região amazônica, considerando o acervo estudado. 


\section{MATERIAL E MÉTODOS}

A obtenção de dados para este estudo baseou-se na pesquisa direta ao banco de dados da Coleção Zoológica Didático-Científica Dr. Joachim Adis, da Universidade do Estado do Pará (CZJA-UEPA). O referido banco de dados detém em suas planilhas o registro de tombo dos espécimes que fazem parte do acervo da coleção, com todas as informações taxonômicas e de procedência, dos exemplares que até o momento se encontram tombados.

Os dados obtidos foram organizados em planilhas e analisados descritivamente, através do programa computacional Microsoft Excel 2007. A elaboração dos mapas de distribuição das espécies utilizou os programas specieslink e geolog para o georreferenciamento das localidades de procedência dos espécimes e o programa Divas-Giz 7.5.0 para a confecção dos mapas de distribuição.

\section{RESULTADOS E DISCUSSÃO}

No acervo da CZJA-UEPA encontram-se registrados no banco de dados 1789 espécimes da Ordem Orthoptera. Esse total encontra-se distribuído em seis superfamílias: Acridoidea $(n=998)$, Proscopioidea $(n=34)$, Pyrgomorphoidea $(n=49)$, Grylloidea $(n=23)$, Tettigonioidea $(n=197)$, Tetrigoidea $(n=40)$ e 444 espécimes que estão identificados apenas na ordem Orthoptera.

A superfamília Acridoidea, alvo deste estudo, está representada no acervo da CZJA-UEPA por duas famílias (Acrididae e Romaleidae), oito subfamílias, 15 tribos, 30 gêneros e 42 espécies (Tabela 1). O número de espécies observadas no acervo da CZJA pode ser considerado relevante, visto que no acervo entomológico da Coleção de Invertebrados do Instituto Nacional de Pesquisas da Amazônia (INPA) que possui mais de 60 anos de existência, se encontram registrados 68 espécies de gafanhotos (INPA, 2016), ou seja, 26 a mais que a CZJA. Essa afirmação se justifica, devido o fato de que a CZJA possui apenas sete anos de existência e conta com uma infraestrutura mínima de incremento, visto que grande parte do seu acervo provém de doações de estudantes e servidores da UEPA.

TABELA 1 - Famílias, subfamílias, tribos, gêneros, espécies e número de exemplares de gafanhotos Acridoidea contidos no acervo da CZJA-UEPA até 2016.

\begin{tabular}{lll}
\hline Família/Subfamília Tribo & Espécie & $\begin{array}{c}\text { Número de } \\
\text { Exemplares }\end{array}$ \\
\hline
\end{tabular}

ACRIDIDAE

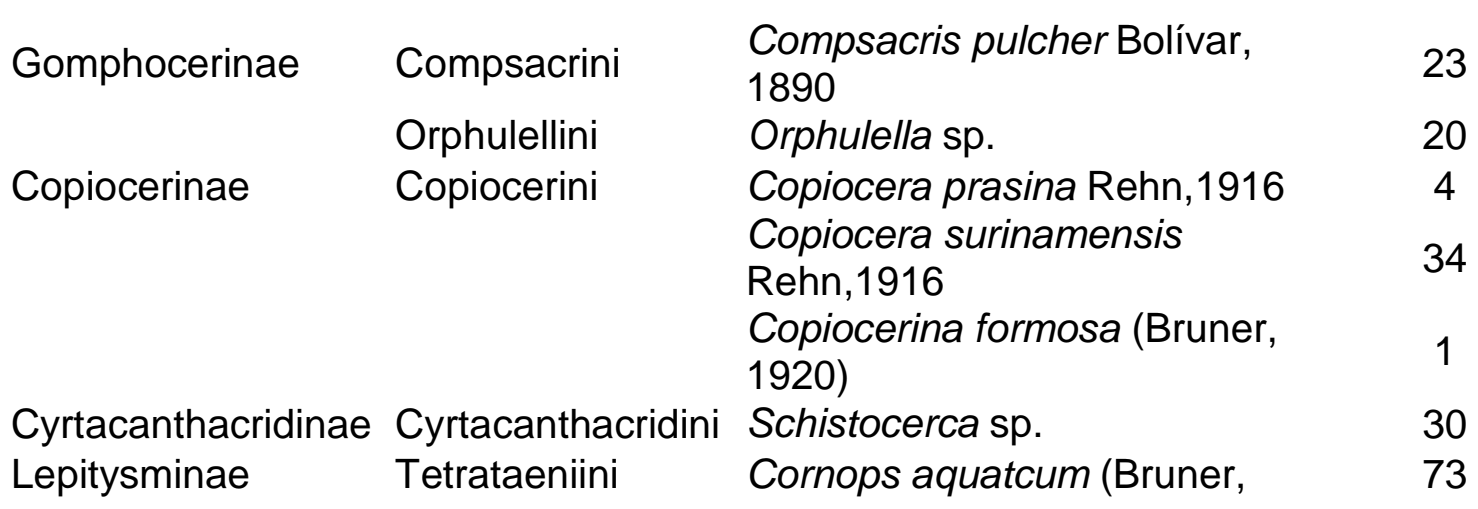

ENCICLOPÉDIA BIOSFERA, Centro Científico Conhecer - Goiânia, v.13 n.24; p.1253 2016 


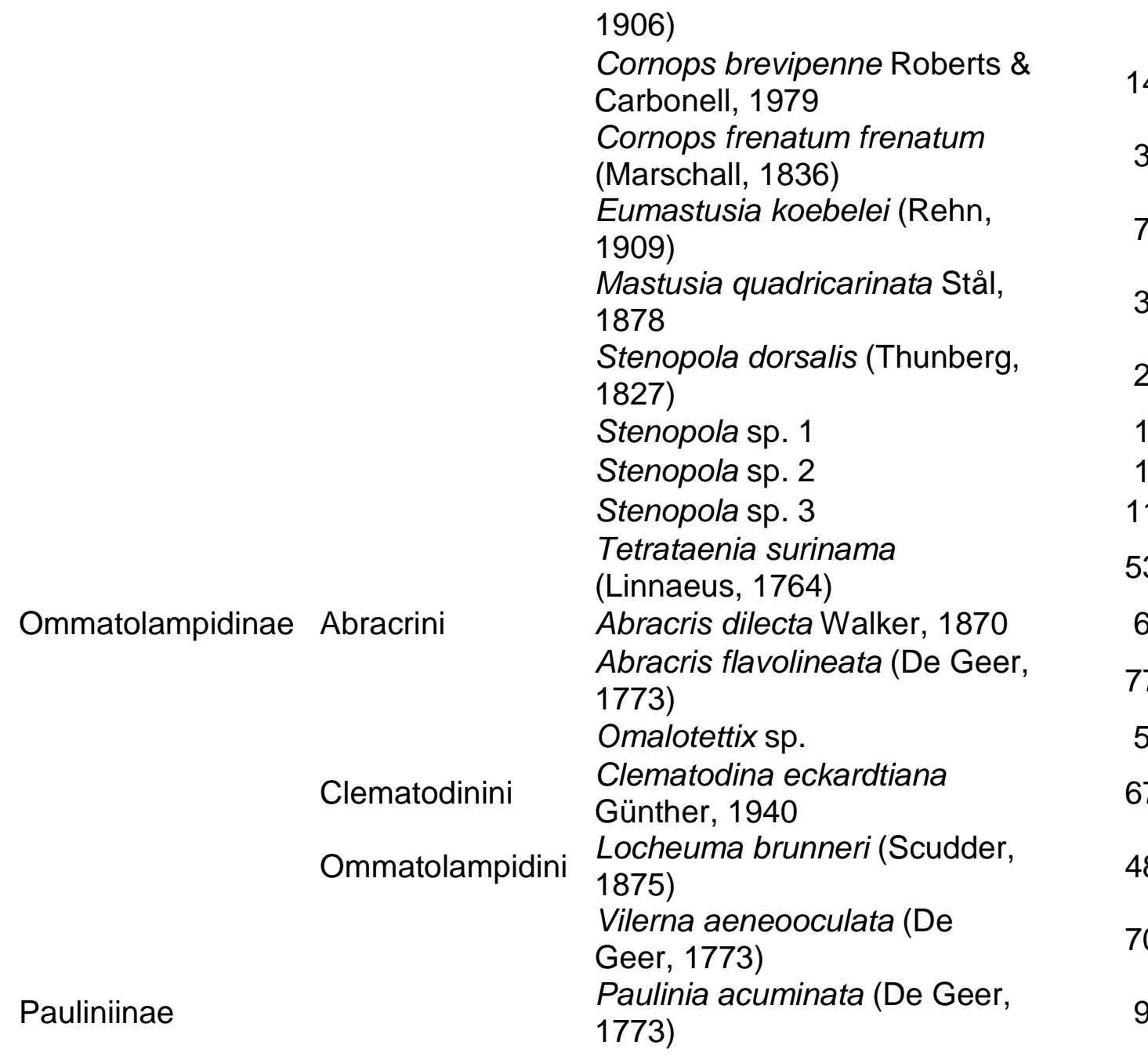

\section{ROMALEIDAE}

Bactrophorinae

Ophthalmolampini

Adrolampis vittagenae (Bruner, 1907)

Adrolampis sp.

Ophthalmolampis sp.

Chariacris dulcis Walker, 1870

Romaleinae

Chariacrini

Prionacris sp

1

Pseudhisychius brasiliensis

Hisychiini

(Bruner, 1911)

Colpolopha obsoleta (Serville,

1831)

Colpolopha sp.

Prionolopha serrata (Linnaeus, 1758)

Procolpia cyanoptera

(Gerstaecker, 1873)

Xyleus discoideus (Serville, 2 
1831)

Phaeoparini

Epiprora hilares Gerstaecker, 1889

Phaeoparia lineaalba

(Linnaeus, 1758)

Romaleini

Chromacris speciosa

(Thunberg, 1824)

Tropidacrini

Titanacris albipes (De Geer, 1773)

Titanacris humboldtii (Scudder, 1869)

Titanacris picticrus picticrus

(Descamps, 1978)

Tropidacris collaris (Stoll,

1813)

Tropidacris cristata cristata (Linnaeus, 1758)

Total 2 / 8

Fonte: os autores (2016).

42 15

Entre as famílias de Acridoidea contidas na CZJA, Romaleidae apresenta o menor número de exemplares $(n=436)$ em relação à família Acrididae que possui o maior número de espécimes $(n=562)$, cujos exemplares pertencem a sete subfamílias: Acridinae, Copiocerinae, Ommatolampidinae, Pauliniinae, Cyrthacanthacridinae, Gomphocerinae e Leptysminae. Destaca-se que embora a família Acrididae tenha uma maior representatividade de espécimes e de subfamílias de gafanhotos no acervo, a subfamília mais abundante pertence à família Romaleidae, cuja espécie Colpolopha obsoleta tem 269 exemplares na coleção (Tabela 1). Entretanto esse número elevado de espécimes de $C$. obsoleta se deve ao fato de que houve coletas de gafanhotos Acridoidea em decorrência de um projeto que foi realizado na Flona de Caxiuanã, de onde os exemplares são oriundos.

Ressalta-se que na Flona de Caxiuanã os ambientes naturais são bem preservados (LISBOA et al., 2013) e, por isso as florestas encontram-se intactas, razão pela qual $C$. obsoleta é abundante, visto que, trata-se de uma espécie esciófila (AMÉDÉGNATO, 2003), ou seja que vive em ambientes sombreados e com boa umidade, como os de florestas primárias ou secundárias, o que também é válido para a espécie Clematodina eckardtiana, que é a quinta espécie mais abundante no acervo da CZJA (Tabela 1).

Quanto às demais espécies da coleção, ressalta-se que sete representam unicatas (um único exemplar) dentro do acervo: Adrolamps sp., Adrolamps vittagenae, Chariacris dulcis e Pseudhiscychius brasiliensis (Romaleidae) e Copiocera formosa, Stenopola sp1., Stenopola sp2 (Acrididae). Entretanto, essa informação não garante que tais espécies sejam raras nos seus locais de procedência (NUNES-GUTJAHR \& BRAGA, 2015), mas pode indicar que tratam-se de espécies que são difíceis de serem coletadas, ou que não parecem ser amplamente distribuídas em seus habitats. 
Ainda quanto às espécies de gafanhotos contidas na CZJA-UEPA, pode-se afirmar que o acervo possui espécies bioindicadoras de qualidade ambiental, tais como C. obsoleta (Romaleidae) e C. eckardtiana (Acrididae) que para se desenvolverem exigem ambientes úmidos e sombreados, com grande quantidade de liteira (folhiço sob o solo) (AMÉDÉGNATO, 2003). Dessa forma, a presença, a maior frequência e a abundância dessas espécies indicam a boa condição estrutural dos ambientes florestados. De modo contrário, a ausência, menor frequência e a menor abundância de tais espécies caracterizam a alteração desses ambientes naturais, principalmente quando se refere ao desflorestamento.

Um destaque especial deve ser dado ao fato de que no acervo da CZJA-UEPA encontram-se nove espécies pertencentes a subfamília Leptysminae $(n=168$ exemplares), a qual possui representantes semiaquáticos (NUNES-GUTJAHR \& BRAGA, 2012). Os gafanhotos semiaquáticos vivem associados a macrófitas aquáticas flutuantes e desempenham papel importante nas cadeias tróficas do meio aquático e semiaquático (NUNES-GUTJAHR \& BRAGA, 2014). Isso se deve ao fato, de que tais gafanhotos fazem parte da dieta alimentar de muitos invertebrados e vertebrados que vivem nas colônias de macrófitas, como sapos, lagartos, aves e peixes, visto que esses gafanhotos são capazes de nadar e mergulhar (NUNES-GUTJAHR \& BRAGA, 2011).

Outro papel importante quanto às espécies de Leptysminae é que algumas delas, como Cornops aquaticum, estão sendo utilizadas como agentes de controle biológico de macrófitas aquáticas (HILL \& OBERHOLZER, 2000, BRAGA et al. 2013). Pois tais plantas quando em ambientes aquáticos alterados, se desenvolvem abundantemente ocasionando sérios danos ao meio aquático, tais como: impedimento do tráfego fluvial, diminuição do volume de água devido a intensa evapotranspiração, baixa de oxigênio, o que pode ocasionar a morte rápida da biota aquática (JUNK, 1980; (BORTOLOTTO \& NETO, 2005)). Além de ocasionar os processos de eutrofização, que prejudicam o meio aquático (BORTOLOTTO \& NETO, 2005).

Outra importância do acervo de Acridoidea da CZJA-UEPA, refere-se à tribo Tropidacrini ( $n=67$ exemplares) que na coleção está representada por cinco espécies: Tropidacris collaris, Tropidacris cristata cristata, Titanacris albipes, Titanacris humboldtii, Titanacris picticrus piticrus (Tabela 1). Estas espécies possuem grande porte, sendo $T$. c. cristata a maior espécie de gafanhotos do mundo (CARBONELL, 1986). As espécies T. c. cristata, T. albipes, T. humboldtii e T. p. piticrus vivem e exploram o dossel de florestas (copas das árvores) e $T$. collaris é considerado um gafanhoto praga de côco, dendê, eucalipto, cana de açúcar, seringa, café, mandioca, além de frutíferas como abacate, banana, manga e plantas ornamentais (BARRIENTOS, 1995; CARBONELL, 1986) e de Acacia mangium (ROMAN et al., 2014). Quanto a isso, é importante ressaltar que no acervo da CZJA existe o registro do gênero Schistocerca (Acrididae: Gomphocerinae) que reúne espécies que possuem potencial para se tornar prega agrícola.

Segundo a classificação do Central for Overseas Pest Research (COPR, 1982) existem, no mundo, nove níveis de dano econômico, que podem ser ocasionados por gafanhotos. Os níveis correspondem as seguintes letras do alfabeto: A, B, C, D, E, F, G, H e K. A letra $\mathbf{A}$ equivale ao nível de maior dano econômico e $\mathbf{K}$ é caracterizado como dano econômico insignificante. Os níveis compreendidos entre as letras A - E justificam a aplicação de medidas de controle. Portanto, é sabido que as espécies do 
gênero Schistocerca, cujos representantes se encontram na CZJA, estão classificadas no nível mais elevado de dano econômico, os quais requerem medidas de controle.

A CZJA desempenha também um importante papel na UEPA por disponibilizar o seu acervo didático para as aulas práticas de biologia dos cursos de graduação. Neste contexto, espécimes de gafanhotos Acridoidea, que não estão tombados, são cedidos para as aulas práticas do Filo Arthropoda, Classe Insecta. Neste sentido, os Acridoidea do acervo também fazem parte das exposições promovidas pela CZJA, as quais correspondem as atividades de popularização da ciência e de divulgação científica e da biodiversidade amazônica, para escolas do ensino básico.

As espécies de Acridoidea contidas no acervo da CZJA-UEPA são oriundas de quatro estados brasileiros: Pará (90,3\%), Maranhão (0,1\%), Amapá $(0,1 \%)$ e Amazonas $(9,5 \%)$. Desses estados, os gafanhotos da coleção são provenientes de municípios, dos quais 19 são do Estado do Pará, três do Amazonas, um município do Amapá e um do Maranhão (Figura 1, Tabela 2). Esse resultado deve-se ao fato de que o maior número de espécies $(n=39)$ e de exemplares $(n=887)$ de gafanhotos são oriundos do Pará e consequentemente de um maior número de localidades e municípios deste estado, o que é contrário ao observado para os estados do Maranhão e Amapá que possuem apenas um gafanhoto oriundo de cada um desses estados (Tabela 2).

TABELA 2 - Estados e número de municípios de procedência e seus respectivos números de famílias, exemplares e espécies de gafanhotos Acridoidea da CZJA-UEPA, até 2016.

\begin{tabular}{ccccc}
\hline Estado & $\begin{array}{c}\text { № de } \\
\text { Municípios }\end{array}$ & $\begin{array}{c}\text { No de } \\
\text { Famílias }\end{array}$ & $\begin{array}{c}\text { № de } \\
\text { Exemplares }\end{array}$ & $\begin{array}{c}\text { № de } \\
\text { Espécies }\end{array}$ \\
\hline Amapá & 1 & 1 & 1 & 1 \\
Amazonas & 3 & 2 & 93 & 8 \\
Maranhão & 1 & 1 & 1 & 1 \\
Pará & 19 & 2 & 887 & 39 \\
\hline
\end{tabular}

Fonte: os autores (2016).

Entre os municípios de procedência dos gafanhotos da CZJA, Portel está representado no acervo por 479 exemplares pertencentes a 16 espécies, seguido de Melgaço com 101 exemplares (21 espécies) e Belém com 94 gafanhotos (18 espécies) (Figura 1). A maior abundância de gafanhotos proveniente de Portel e Melgaço (arquipélago do Marajó) se deve ao desenvolvido um projeto de Biodiversidade que proporcionou a realização de inventários faunísticos, nos quais pesquisadores da CZJA participaram, devido uma parceria com o Museu Paraense Emílio Goeldi (MPEG), que disponibilizou, como base de pesquisa a Estação Científica Ferreira Penna (ECFPn), na Floresta Nacional de Caxiuanã. A área da Flona de Caxiuanã abrange os municípios de Melgaço e Portel, respectivamente, o que possibilitou as coletas de gafanhotos Acridoidea em ambos os municípios.

Quanto aos exemplares oriundos de Belém, ressalta-se que este é o município sede do Estado do Pará, onde está situada a UEPA e consequentemente a CZJA. Deve-se destacar que os gafanhotos oriundos deste município, no acervo, são 
provenientes de doações feitas por estudantes e servidores da universidade, que residem neste município. Por tal razão, os gafanhotos pertencem a espécies mais adaptadas aos ambientes antropizados, como o próprio meio urbano, onde esses insetos exploram os quintais e jardins de residências, praças e outros espaços com vegetação na cidade. Quanto a isso, os gafanhotos que exploram os locais mencionados, pertencem às espécies consideradas invasoras, que possuem hábito generalista (NUNES-GUTJAHR \& BRAGA, 2015). Por tal razão, tais espécies, apresentam ampla distribuição geográfica e ambiental, como é o caso do Acridídeo Abracris flavolineata (AMÉDÉGNATO, 2003).

Considerando outros acervos amazônicos, destacam-se as coleções de Invertebrados do Instituto Nacional de Pesquisas da Amazônia (INPA) que detém um acervo de Acridoidea com 2144 exemplares pertencentes a 165 espécies (http://colecoes.inpa.gov.br/invertebrados/html/principais/bancodedados.php) e a coleção de Invertebrados do Museu Paraense Emílio Goeldi (MPEG) que reúne 3284 exemplares com 197 espécies de gafanhotos Acridoidea (BRAGA, 2010). Ressalta-se que estas coleções são antigas (INPA tem 62 anos) e seculares (MPEG tem 150 anos), o que justifica a quantidade de exemplares e de espécies de Acridoidea em seus acervos. Quanto a isso, embora a CZJA-UEPA seja uma coleção jovem (7 anos), que possui, ainda, um acervo pequeno de gafanhotos, deve-se destacar que este corresponde a $46,5 \%$ do número de exemplares da coleção do INPA e $30,4 \%$ da coleção do MPEG e a $25,5 \%$ do número de espécies da coleção do INPA e $21,3 \%$ das espécies de gafanhotos contidas no MPEG. Apesar disso, é relevante considerar a importância da existência do acervo da CZJA, que é exclusivamente amazônico e que, embora pequeno, detém uma amostra dos testemunhos da biodiversidade dessa região.

Quanto ao exposto, pode-se considerar que na situação atual, o acervo de gafanhotos verdadeiros da CZJA-UEPA reúne espécies que exploram ambientes naturais preservados, antropizados e urbanos e que já pode contribuir para gerar informações sobre a biodiversidade amazônica. Entretanto, o nível de representatividade desses insetos no acervo, indica uma deficiência, quanto aos municípios e principalmente aos estados amazônicos, visto que apenas quatro destes estão representados no acervo de Acridoidea da CZJA. Contudo, deve-se valorizar a CZJA, não somente pelo que representa e possui, mas pelo fato de que os gafanhotos Acridoidea dessa coleção se encontrarem catalogados, tombados e em bom nível de aferimento taxonômico, podendo contribuir ou possibilitar o desenvolvimento de estudos de diferentes áreas do conhecimento.

Os resultados apresentados neste estudo, evidenciam que os espécimes e consequentemente as espécies de Acridoidea da CZJA são provenientes de poucas localidades da região amazônica, sendo em grande parte da região metropolitana de Belém e dos municípios de Melgaço e Portel, o que ocasiona uma concentração de pontos no mapa de procedência das espécies (Figura 1). Isso indica que o acervo de Acridoidea na CZJA-UEPA necessita de expansão e principalmente da realização de inventários direcionados para outros estados e localidades da região amazônica, os quais possam promover o incremento da referida coleção. 


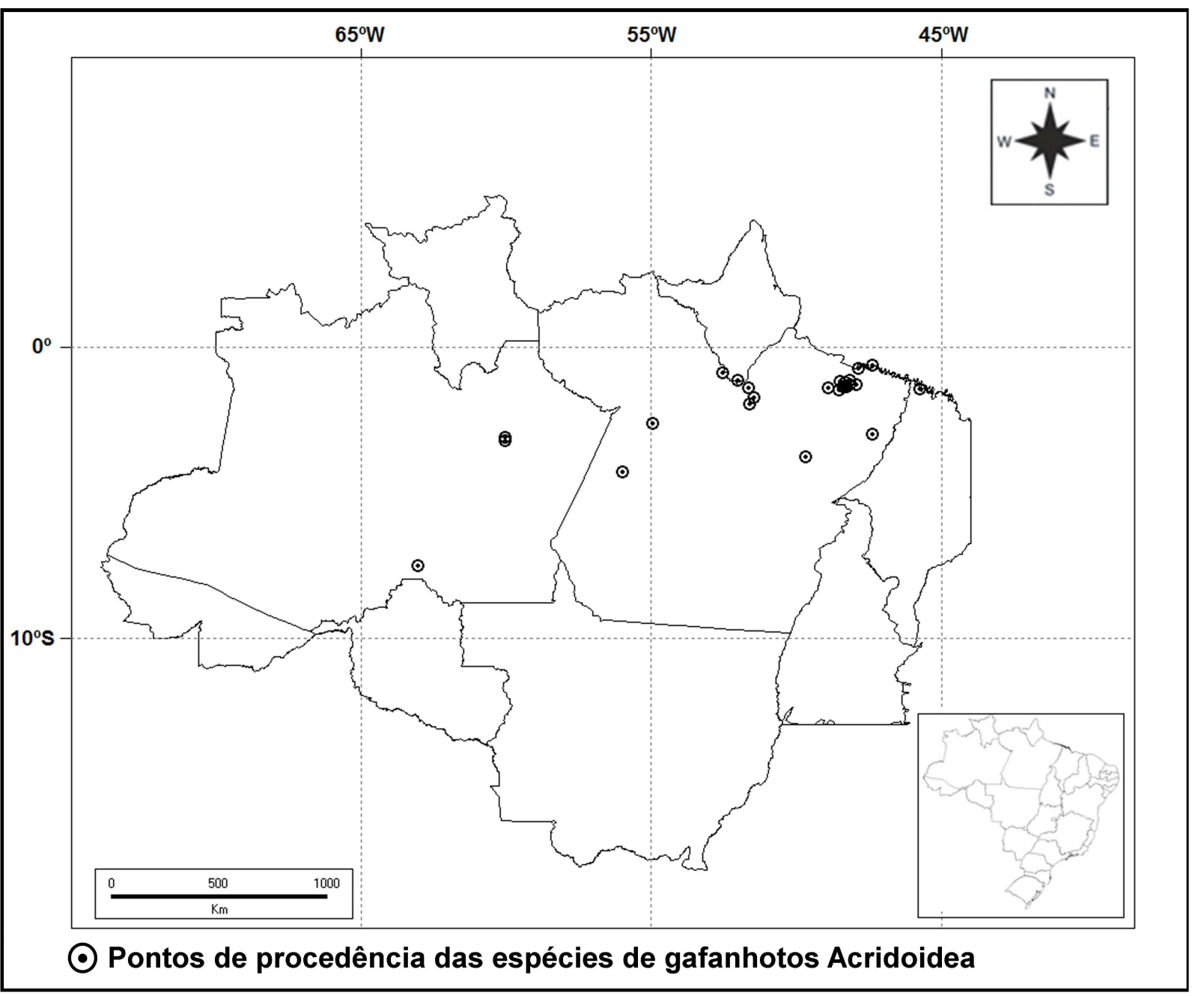

FIGURA 1 Pontos de procedência das espécies de gafanhotos Acridoidea presentes no acervo da Coleção Zoológica Didático-Científica Dr. Joachim Adis da UEPA, nos Estados do Amapá, Amazonas, Pará e Maranhão, na Amazônia Legal. Fonte: os autores (2016).

\section{CONCLUSÃO}

A Coleção Zoológica Didático-Científica Dr. Joachim Adis, da Universidade do Estado dos Pará, reúne em seu acervo, até o momento, espécies de gafanhotos Acridoidea exclusivamente amazônicos. Este acervo ainda está em formação, embora guarde espécies que exploram ambientes naturais preservados, antropizados e urbanos. Ressalta-se, quanto a isso, que neste acervo as espécies urbanas são, principalmente, oriundas da cidade de Belém-PA, onde se encontra situada a CZJA.

$O$ acervo de gafanhotos Acridoidea da CZJA ainda é pequeno, suas espécies pertencem a gafanhotos terrestres e semiaquáticos, sendo que algumas destas são bioindicadoras de qualidade Ambiental (Colpolopha obsoleta e Clematodina eckarditiana) e controladoras biológicas de macrófitas aquáticas (Cornops aquaticum). 
A CZJA é uma coleção jovem, e por tanto é aceitável que seu acervo seja ainda pouco expressivo quanto ao número de exemplares e de espécies, embora se deva considerar que os acervos de coleções somente se desenvolvem quando existem pesquisadores dedicados e principalmente taxônomos para realizar a identificação do material zoológico acondicionado. Todos os espécimes da coleção encontram-se catalogados e tombados em banco de dados, para que em breve, todas as informações de tais insetos sejam disponibilizadas na rede mundial de computadores, a fim de difundir informações dos gafanhotos que representam parte da biodiversidade amazônica.

\section{REFERÊNCIAS}

AMÉDÉGNATO, C. Microhabitat distribution of forest grasshoppers in the Amazon. In: BASSET, Y.; NOVOTNY, V.; MILLER, S. E.; KITCHING, R. L. Arthropods of tropical forests: spatio-temporal dynamics and resource use in the canopy. Cambridge University Press, Cambridge, 2003, p. 237-255.

AFONSO, R.; LEMES, P. G.; SARMENTO, R. A.; LEITE, P. J. B.; NETO, M. P.; ANJOS, N. First Report of Giant Grasshopper Tropidacris collaris (Orthoptera: Acridoidea: Romaleidae) Attacking Plantations of Acacia mangium (Fabaceae) in Brazil. Journal of the Kansas Entomological Society, v. 87, n. 1, p. 102-105, 2014. doi: http://dx.doi.org/10.2317/JKES130408.1

AURICCHIO, P.; SALOMÃO, M. G. Técnicas de coleta e preparação de vertebrados para fins científicos e didáticos. Instituto Pau Brasil de História Natura, São Paulo, Aruja, 2002.

BARRIENTOS, L. L. The present state of the locust and grasshopper problem in Brazil. Jornal of Orthoptera Research, v. 4, p. 61-64, 1995.

BORTOLOTTO, I. M.; NETO, G. G. O uso do camalote, Eichhornia crassipes (Mart. Solms), Pontederiaceae, para confecção de artesanato no Distrito de Albuquerque, Corumbá, MS, Brasil. Acta Botanica Brasilica, v. 19, n. 2, p. 331-337, 2005.

BRAGA, C. E. Mapeamento e Análise da Distribuição de Orthopteroidea: Ordens Orthoptera (Acridoidea), Phasmatodea e Blattodea da Coleção de Invertebrados do Museu Paraense Emílio Goeldi. PCI/MCT/MPEG, 2010.

BRAGA, C. E.; NUNES-GUTJAHR, A. L.; MORAIS, J. W.; ADIS, J. Avaliação do potencial do gafanhoto Cornops aquaticum (Orthoptera) como agente de controle Biológico de Eichhornia crassipes (Pontederiaceae). Interciencia (Caracas), v. 38, p. 590 - 596, 2013.

CARBONELL, C. S. Revision of the Neotropical Genus Tropidacris (Orthoptera, Acridoidea, Romaleidae, Romaleinae). Proceedings of the Academy of Natural Sciences of Philadelphia, v. 138, n. 2, p. 366-402, 1986. 
C.O.P.R. (Centre for Overseas Pest Research). The locust and grasshopper agricultural manual. London, 1982.

GUEDES, M. L. P. Culicidae (Diptera) no Brasil: relações entre diversidade, distribuição e enfermidades. Oecologia Australis, v. 16, n. 2, p. 283-296, 2012. Disponível em: <http://dx.doi.org/10.4257/oeco.2012.1602.07>. doi: 10.4257/oeco.2012.1602.07.

HADDAD, C. F. B. Biodiversidade de anfíbios no estado de São Paulo. In: JOLY, C. A.; BICUDO, C. E. de M. (orgs.) Biodiversidade do estado de São Paulo: síntese do conhecimento ao final do século XX. v. 6: Vertebrados. São Paulo. Fapesp, 1998, 15-26 p.

HILL, M. P.; OBERHOLZER, I. G. Host specifity of the grasshorpper, Cornops aquaticum a natural enemy of water hyacinth. In: Neal R. Spencer. Proceedings of the $X$ International Symposium on Biological Control of Weeds. Bozeman, Montan. p. 349-356, 2000.

INSTITUTO NACIONAL DE PESQUISAS DA AMAZÔNIA - INPA. Coleções de Invertebrados: banco de dados. Disponível em: $<$ http://colecoes.inpa.gov.br/invertebrados/html/principais/bancodedados.php $>$ Acesso em: 03 de out. 2016.

JUNK, W.J. Áreas inundáveis - Um desafio para limnologia. Acta Amazonica, v. 10, n. 4, p. $775-795,1980$.

LISBOA, P. B. Caxiuanã: paraíso ainda preservado. Museu Paraense Emílio Goeldi, Belém, 2013.

MAGALHÃES, C; SANTOS, J. L. C.; SALEM, J. I. Automação de coleções biológicas e informações sobre a biodiversidade da Amazônia. Parcerias estratégicas, v. 12, p. 294-312,2001.

http://seer.cgee.org.br/index.php/parcerias estrategicas/article/viewFile/184/178

NUNES-GUTJAHR, A. L.; BRAGA, C. E. Gafanhotos. In: Oliveira, M. L., Bacaro, F.B., Braga-Neto, R., Magnusson, W. E. Reserva Ducke: A Biodiversidade Amazônica através de uma grade. Org. Editora INPA. Manaus - AM, 2011.

NUNES-GUTJAHR, A. L.; BRAGA, C. E. Gafanhotos da Flona Caxiuanã: Guia prático. Museu Paraense Emilio Goeldi, Belém, 2012.

NUNES-GUTJAHR, A. L.; BRAGA, C. E. Ordem Orthoptera In: Hamada, N.; Nessimian, J. L.; Querino, R. B. Insetos aquáticos na Amazônia brasileira: taxonomia, biologia e ecologia. 1 ed. Manaus: Editora do INPA, 2014. 297-312p.

NUNES-GUTJAHR, A. L.; BRAGA, C. E. Análise faunística de gafanhotos Acridoidea da Volta Grande do Rio Xingu, área de influência direta da Hidrelétrica Belo Monte, 
Pará, Brasil. Ciência Rural, v. 45, n. 7, p. 1220-1227, 2015. Disponível em DOI: <http://dx.doi.org/10.1590/0103-8478cr20120442>.doi:10.1590/0103-8478cr20120442

PAPAVERO, N. Fundamentos Práticos de Taxonomia Zoológica: coleções, bibliografia, nomenclatura. São Paulo: Ed. Univ. Estadual Paulista, 1994.

RIBEIRO, E. S. Museus em universidades públicas: entre o campo científico, o ensino, a pesquisa e a extensão. Museologia \& Interdisciplinaridade, v. 1, n. 4, 2013.

SILVA, M. C.; RIVERO, A. A. C.; SALLES, L. O. Musealização do acervo do Museu de Zoologia da Universidade de São Paulo: salvaguarda e comunicação de cinco mamíferos taxidermizados da fauna brasileira. Revista CPC, n. 13, p. 74-106, 2012. http://www.revistas.usp.br/cpc/article/view/15690/17264.

SOUZA, N. S. A Amazônia brasileira: processo de ocupação e a devastação da floresta. Boletim Científico ESMPU, v. 9, n. 32/33, p. 199-235, 2010.

UNITED NATIONS ENVIRONMENT PROGRAMME - UNEP. An assessment of the status of the World's Remaining Closed Forests. s.1.: UNEP/DEWA/TR, Washington, District of Columbia, 2001. 